\title{
Self-standing chitosan films as dielectrics in organic thin-film transistors
}

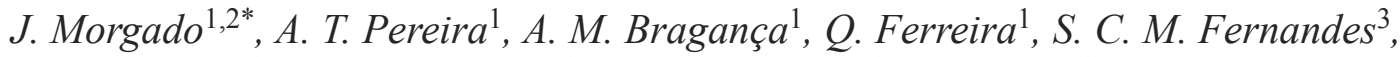 \\ C. S. R. Freire ${ }^{3}$, A. J. D. Silvestre ${ }^{3}$, C. Pascoal Neto ${ }^{3}$, L. Alcácer ${ }^{1}$ \\ ${ }^{1}$ Instituto de Telecomunicações, Instituto Superior Técnico, Avenida Rovisco Pais, P-1049-001 Lisboa, Portugal \\ ${ }^{2}$ Department of Bioengineering, Instituto Superior Técnico, UTL, Avenida Rovisco Pais, P-1049-001 Lisboa, Portugal \\ ${ }^{3}$ CICECO, University of Aveiro, Campus de Santiago, P-3810-193 Aveiro, Portugal
}

Received 26 June 2013; accepted in revised form 9 August 2013

\begin{abstract}
Organic thin film transistors, using self-standing $50 \mu \mathrm{m}$ thick chitosan films as dielectric, are fabricated using sublimed pentacene or two conjugated polymers deposited by spin coating as semiconductors. Field-effect mobilities are found to be similar to values obtained with other dielectrics and, in the case of pentacene, a value $(0.13 \mathrm{~cm} /(\mathrm{V} \cdot \mathrm{s}) \mathrm{compara}-$ ble to high performing transistors was determined. In spite of the low On/Off ratios (a maximum value of 600 was obtained for the pentacene-based transistors), these are promising results for the area of sustainable organic electronics in general and for biocompatible electronics in particular.
\end{abstract}

Keywords: biocompatible polymers, organic thin-film transistors, organic field-effect transistors, chitosan, dielectric

\section{Introduction}

There is a significant effort being put into the development of organic electronics employing various renewable materials, encompassing paper, leather, silk and biodegradable plastics, for various functions [1]. There are very interesting possibilities in terms of the development of 'green', biocompatible and biodegradable electronics with low production costs. For instance, various researches are being focused on paper. This has been studied as a substrate for printed circuits, using low cost fabrication methods. The fact that these systems combine flexibility and lightweight opens up various areas of application such as consumer electronics, disposable systems and applications in medical sensing and micro-electromechanical systems [2]. Paper was also used as material for flexible electronics [3].

Chitosan (CS) is a cationic polysaccharide obtained by alkaline deacetylation of chitin, this being the second most abundant polymer in nature, after cellulose. Chitin appears in nature as ordered crystalline microfibrils forming structural components of the exoskeleton of crustacean and insects and in the cell walls of fungi and algae $[4,5]$. In view of its properties, like excellent film-forming ability, biocompatibility and biodegradability [4] chitosan and derivatives have been used in biomedical applications, such as gene delivery, biosensors [6] and also as proton exchange membranes [7]. These applications offer the prospects for the fabrication of biocompatible devices that could operate in biologically relevant media (for instance for smart sensing and preventive medical care), an area that is attracting an increasing attention [8].

In 2011, two reports appeared on the use of chitosan-based materials in electronics. One paper, by Zhong et al. [9], explored the protonic conduction in maleic-chitosan thin films to fabricate a protonic

\footnotetext{
${ }^{*}$ Corresponding author, e-mail: jorge.morgado@1x.it.pt (C) BME-PT
} 
field-effect transistor, yielding a proton mobility of $4.9 \cdot 10^{-3} \mathrm{~cm}^{2} /(\mathrm{V} \cdot \mathrm{s})$. In another study, chitosan was used as dielectric in an ITO-based thin film transistor [10]. In this study, a chitosan film was deposited on ITO-coated glass by drop cast from an acetic acid solution. No indication of its thickness was given. ITO source, drain and channel were fabricated by RF magnetron sputtering. The devices were tested at ambient conditions. The authors obtained high performance transistors, which they attribute to the influence of proton mobility leading to very high gate dielectric capacitance due to electric-double-layer effect at the interface between dielectric and the semiconducting oxide. No reports were found on the use of CS as dielectric material in organic transistors.

In view of the CS potential, we have been studying self-standing CS films both as substrates and dielectrics for organic thin film transistors (TFTs). Here, we report on the performance of TFTs fabricated with organic semiconductors deposited by sublimation (pentacene) and by spin coating (regioregular head-to-tail poly(3-hexylthiophene), P3HT, and poly(9,9-dioctylfluorene-alt-bithiophene), F8T2), on the bare surface of self-standing CS films.

We find high hole field-effect mobilities (peaking at $0.13 \mathrm{~cm}^{2} /(\mathrm{V} \cdot \mathrm{s})$ for pentacene) though the On/Off ratios are small. A strong hysteresis effect is found which is attributed to the presence of ionic mobile species in the CS films.

\section{Experimental}

Chitosan, with a degree of deacetylation of $97 \%$ and a viscosity-average molar mass of $350000 \mathrm{~g} / \mathrm{mol}$, was purchased from Mahtani Chitosan Pvt. Ltd. (India) and purified before use [11]. For the preparation of the CS films, a solution $1.5 \%(\mathrm{w} / \mathrm{v})$ at $\mathrm{pH}=4$ was first prepared by dissolving the purified powdered CS sample in aqueous acetic acid $(1 \% \mathrm{v} / \mathrm{v})$ at room temperature during $48 \mathrm{~h}$. An appropriate mass of CS solution was then transferred onto $10 \times 10 \mathrm{~cm}^{2}$ square plates. $\sim 50 \mu \mathrm{m}$ thick films were obtained by casting at $30^{\circ} \mathrm{C}$ in a ventilated oven for $16 \mathrm{~h}$. The obtained films were kept in atmospheric conditions until they were used for the thin film transistors fabrication.

The surface of the CS films were characterized by atomic force microscopy, using a Molecular Imag- ing Agilent (model 5100) system, operating in noncontact mode (at a resonance frequency between 200 and $400 \mathrm{kHz}$ ) using silicon probes with tip radii lower than $10 \mathrm{~nm}$.

Thin-film transistors were prepared by depositing, first, a gate contact of ca. $40 \mathrm{~nm}$ thick aluminum on one (down) side of the CS film, using an Edwards evaporator at a base pressure of ca. $2 \cdot 10^{-5}$ mbar. Pentacene-based TFTs were prepared by thermal deposition depositing, on the other side of the CS film, a ca. $150 \mathrm{~nm}$ thick pentacene film. Transistors with F8T2 were prepared in air by spin coating from a xylene solution. A thin F8T2 film (ca. $100 \mathrm{~nm}$ thick) was thus formed on the CS film surface, opposite to the $\mathrm{Al}$ gate contact. These two types of device structures (with pentacene and F8T2) were then transferred inside a glove box $\left(<0.1 \mathrm{ppm} \mathrm{O}_{2}\right.$, $<0.3 \mathrm{ppm} \mathrm{H}_{2} \mathrm{O}$ ) to deposit source and drain contacts. P3HT was deposited by spin coating inside the glove box. The film thickness is ca. $40 \mathrm{~nm}$. These three types of thin film transistors were then finalized by depositing source and drain contacts through a shadow mask, defining a channel with a length of $L=100 \mu \mathrm{m}$ and a width of $W=5.1 \mathrm{~mm}$. Source and drain contacts consisted of a bilayer structure, comprising $\mathrm{MoO}_{3}$ (ca. $20 \mathrm{~nm}$ thick, which is a very efficient hole-injection material) covered with a 50$60 \mathrm{~nm}$ thick layer of Al. These transistors were characterized inside the glove box (to avoid damaging the source and drain contacts upon contact with ambient humidity), 24-48 h after the source and drain deposition, using a Semiconductor Parameter Analyzer HP 4140B. We note that the three types of devices were prepared from the same large CS film. We therefore expect that the characteristics of the CS dielectric, namely the water content, are similar for all three TFT structures. The thicknesses of the organic semiconductors were determined in films deposited, under similar conditions, on a glass substrate, using a Dektak 6M profilometer.

The specific capacitance of the CS film was measured with an Impedance Analyzer HP4192A at $1 \mathrm{kHz}$. A capacitor structure was fabricated by ink jet printing silver of both sides of the CS film, using a Dimatix printer.

\section{Results and discussion}

Figure 1 shows the topography image obtained over an area of $10 \times 10 \mu \mathrm{m}^{2}$. Some fibers with diameters 


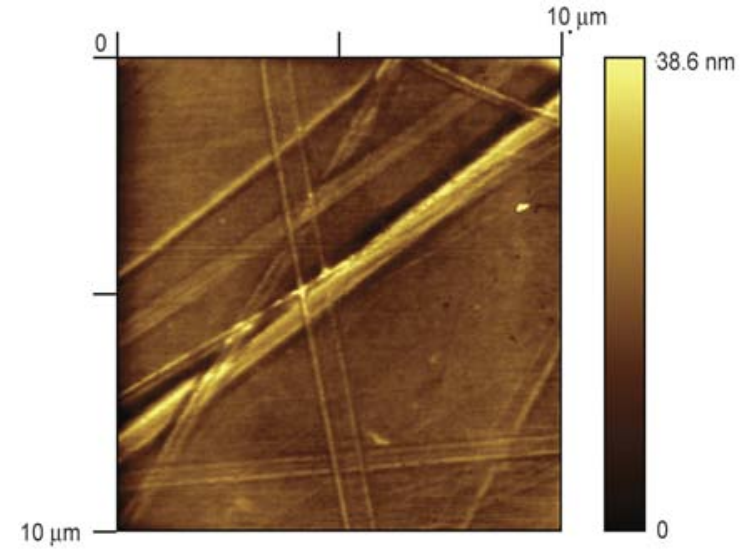

Figure 1. Topography image of a CS film over an area of $10 \cdot 10 \mu \mathrm{m}^{2}$, evidencing some fibers dispersed over a very regular surface

on the order of $150 \mathrm{~nm}$ are observed. The regions between the fibers appear very regular, with a rootmean-square roughness $\left(R_{\mathrm{rms}}\right)$ not exceeding $1 \mathrm{~nm}$. The specific capacitance of the CS film measured for the capacitor structure fabricated by ink jet printing silver of both sides of the CS film at $1 \mathrm{kHz}$ is $C_{0}=9.54 \cdot 10^{-9} \mathrm{~F} / \mathrm{cm}^{2}$. We note that, a lower capacitance value of $3.26 \cdot 10^{-11} \mathrm{~F} / \mathrm{cm}^{2}$ was measured, also at $1 \mathrm{kHz}$, when the $\mathrm{CS}$ film is placed in between two gold discs. We attribute this difference to the porous nature of the CS films surface, leading to an effective thickness that is much lower than the 'geometrical' value considered when using the gold discs. Although Figure 1 evidences a flat surface, a detailed study over smaller areas, confirms the existence of a porous structure, as shown in Figure 2. Though the line profile shows that such pores are few nanometers deep. However, this is likely limited by the tip radius, thus preventing us to determine the real value.

We further note that a comparable specific capacitance of ca. $10^{-7} \mathrm{~F} / \mathrm{cm}^{2}$ was determined at $1 \mathrm{kHz}$ in a drop cast film of chitosan on ITO, though the film thickness was not reported [10]. It should be pointed out that, in view of its highly hygroscopic nature, the dielectric response of the chitosan is dependent on the water content [12] and likely on the deacetylation degree, leading to variable values in different reports.

Figure 3 shows the output characteristics and the transfer curve for the pentacene-based TFT. The curves are typical of a p-type TFT working in accumulation, as typically found in pentacene-based TFTs. The output curves were recorded at two different sweep rates. We find that a lower sweep rate

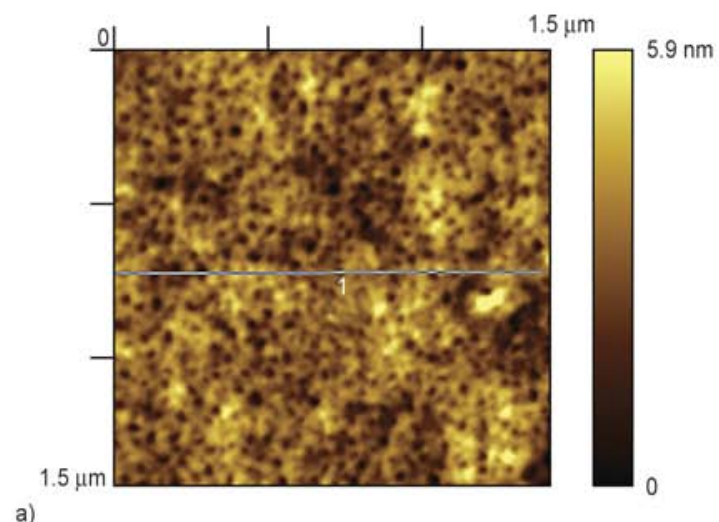

a)

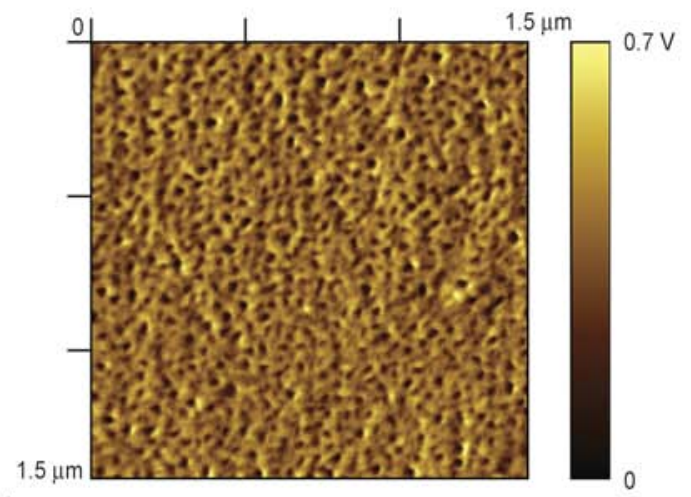

b)

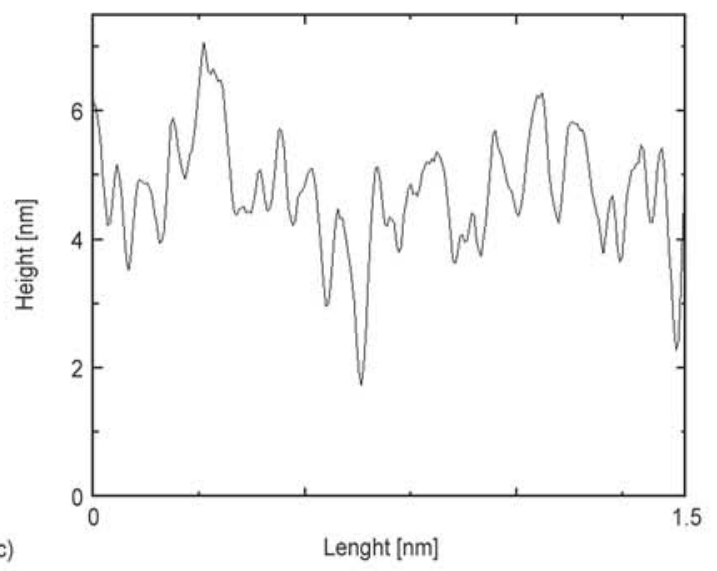

Figure 2. a) Topography and b) phase image of a CS film over an area of $1.5 \times 1.5 \mu \mathrm{m}^{2}$, evidencing the porous structure. c) Line profile corresponding to the line marked in a).

leads to slightly higher currents. The transfer curve exhibits a marked hysteresis, which is attributed to mobile ions within the chitosan dielectric [10]. This hysteresis, with an increase of the current in the reverse sweep, is consistent with the output current increase upon decrease of the sweep rate (Figure 3a) and attributed to ion displacements within CS film. The transfer curve shows a low On/Off current ratio (between $V_{\mathrm{g}}=-20$ and $V_{\mathrm{g}}=0 \mathrm{~V}$ ) of about 70 (in the forward sweep), which is reduced to ca. 4 in the back sweep. From the linear region of the $\left(I_{\mathrm{d}}\right)^{1 / 2} v s$ 

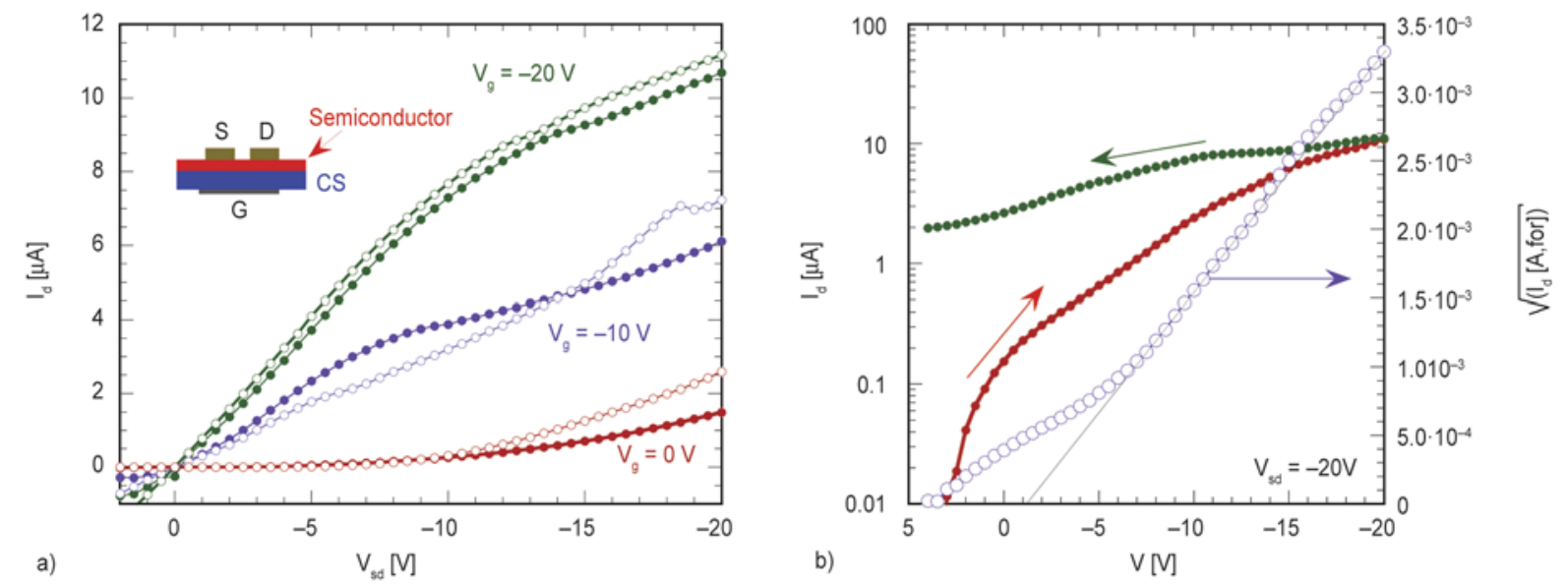

Figure 3. Output and transfer curves for a pentacene TFT. a) Two measuring rates were used, $0.5 \mathrm{~V} / \mathrm{s}$ (closed symbols) and $0.5 \mathrm{~V} / 5 \mathrm{~s}$ (open symbols). The inset shows the TFT scheme. The transfer curve (b) was measured at rate of $0.5 \mathrm{~V} / \mathrm{s}$. In b) we also show the plot of $\left(I_{\mathrm{d}}\right)^{1 / 2}$ as a function of the gate voltage $\left(V_{\mathrm{g}}\right)$ for the forward ( +4 to $\left.-20 \mathrm{~V}\right)$ sweep.

$V_{\mathrm{g}}$ plot, we estimate a saturation mobility of $0.13 \mathrm{~cm}^{2} /(\mathrm{V} \cdot \mathrm{s})$, using the well-known relation given by Equation (1):

$I_{\mathrm{d}}=\mu \frac{W C_{0}}{2 L}\left(V_{\mathrm{g}}-V_{\mathrm{th}}\right)^{2}$

Figure 4 shows the transfer curves for TFTs based on F8T2 and P3HT prepared by spin coating. Both TFTs show the typical p-type behavior. F8T2-based TFTs exhibit the highest On/Off ratio of ca. 600 (in the forward sweep), reduced to 60 in the back sweep, while the values found for the P3HT-based TFTs are ca. 6 and 3 respectively. The saturation field-effect mobility determined for the P3HTbased TFT is $8.6 \cdot 10^{-3} \mathrm{~cm}^{2} /(\mathrm{V} \cdot \mathrm{s})$. In the case of the F8T2-based TFT, if we just fit the data for $V_{\mathrm{g}}$ between -15 and $-20 \mathrm{~V}$, where the slope of the $\left(I_{\mathrm{d}}\right)^{1 / 2}$ vs $V_{\mathrm{g}}$ curve has decreased, we obtain a fieldeffect mobility of $9 \cdot 10^{-4} \mathrm{~cm}^{2} /(\mathrm{V} \cdot \mathrm{s})$.

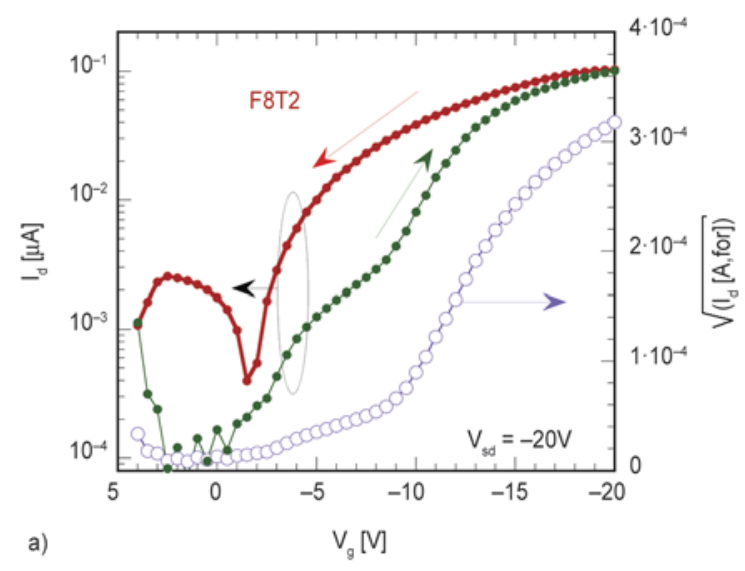

Figure 4. Transfer curves of TFTs based on F8T2 (a) and P3HT (b). The arrows indicate the voltage sweep. Also shown is $\left(I_{\mathrm{d}}\right)^{1 / 2}$ vs $V_{\mathrm{g}}$ (open symbols).
These results show that CS dielectric allows the formation of a conducting channel with these three organic semiconductors. The On/Off ratios are very small, but the mobilities are close to the highest values reported for other dielectrics. For instance, a mobility of $0.4 \mathrm{~cm}^{2} /(\mathrm{V} \cdot \mathrm{s})$ was reported for pentacene TFTs with a silane-modified $\mathrm{SiO}_{2}$ dielectric and $\mathrm{MoO}_{3} / \mathrm{Al}$ source and drain contacts [13]. We note that, for similarly prepared TFTs with $625 \mathrm{~nm}$ thick $\mathrm{SiO}_{2}$ dielectric, we obtained a mobility of ca. $8 \cdot 10^{-4} \mathrm{~cm}^{2} /(\mathrm{V} \cdot \mathrm{s})$ for P3HT, which is lower than the value obtained with CS dielectric. However, this is also much lower than the values reported for primed P3HT transistors (e.g. $0.12 \mathrm{~cm}^{2} /(\mathrm{V} \cdot \mathrm{s})[14]$ ). Finally, mobilities on the order of $10^{-2} \mathrm{~cm}^{2} /(\mathrm{V} \cdot \mathrm{s})$ were reported for optimized TFTs structures based on aligned F8T2 [15]. It should be emphasized that no surface treatment of the CS film and no thermal annealing was carried out on the organic semicon-

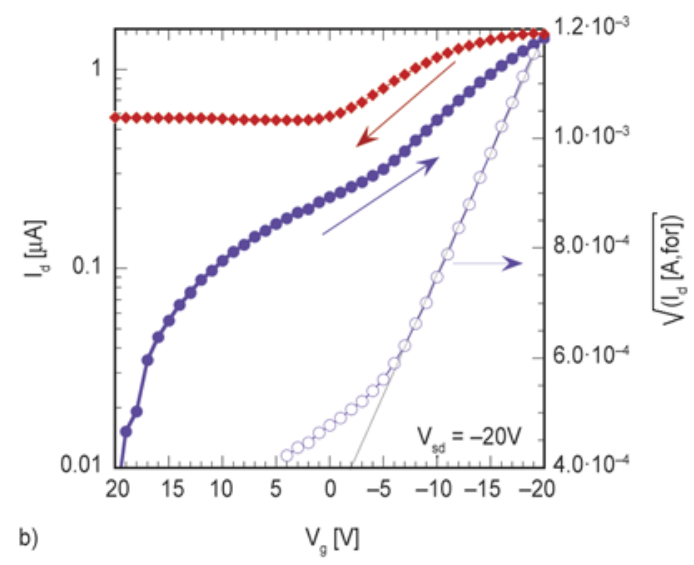



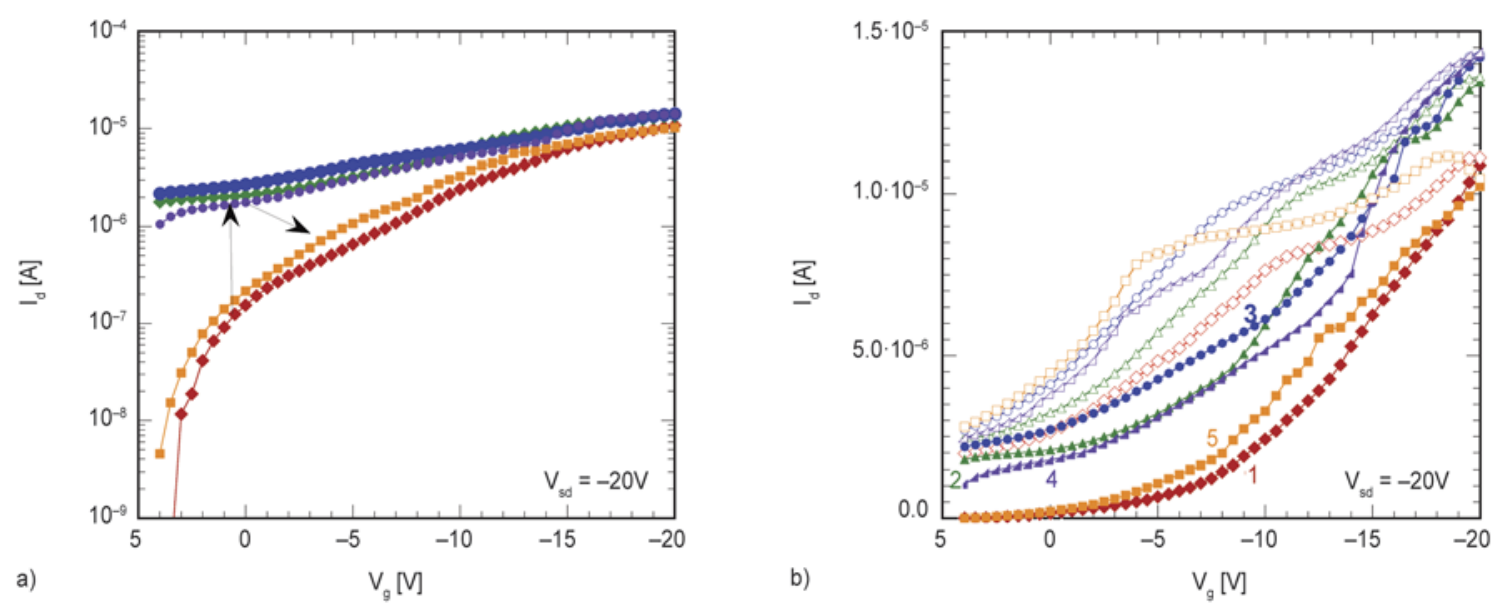

Figure 5. Forward transfer curves for the pentacene-based TFT of sequential sweep cycles. Measurements were carried out at a sweep rate of $0.5 \mathrm{~V} / \mathrm{s}$, starting at curve 1 up to 4 . Curve 5 was obtained after ca. $2 \mathrm{~h}$ resting time, evidencing an almost complete recovery of the initial behavior. A different color and symbol type is associated to each cycle.

ducting films. We therefore anticipate that there is still room for improvement of the performance here reported. The other significant characteristic of the CS TFTs is the presence of significant hysteresis. Figure 5 shows the effect of the hysteresis on the transfer curve of pentacene-based transistors on five sequential voltage cycles. Figure 3 showed that, the Off current in the back sweep is much higher than that measured in the forward sweep. Figure 5a shows the transfer curves obtained in the forward sweep of each cycle, along 4 sequential cycles. The measured curve remains relatively constant between the $2^{\text {nd }}$ and $4^{\text {th }}$ cycles. If the TFT is left to recover for two hours we obtain a transfer curve very similar to the first one. The complete voltage cycles are shown in Figure 5b, where the forward sweep corresponds to the filled symbols and the back sweep to the open symbols. We attribute these results to ionic displacements within the CS dielectric in response to the applied gate bias.

It was mentioned above that $\mathrm{CS}$ is a hygroscopic material and that its proton conductivity has indeed been explored in previous studies. The effect of the water content was not addressed here but, based on the studies carried out by Kaihovirta et al. [16], for instance, in TFTs with hygroscopic dielectrics, the water content may in fact play an important role on the performance of transistors. Additional studies are in progress to better evaluate the role of the water content and the possible electric-double-layer effects on the behavior of the organic transistors.

\section{Conclusions}

In summary, we showed that self-standing chitosan films are very interesting dielectrics for organic TFTs. Transistors prepared with either thermal evaporated or spin coated films of organic semiconductors showed high hole field-effect mobilities though the On/Off ratios are small. We believe these studies open the way for the application of CS films in organic electronics for a wide range of applications, ranging from disposable systems to biosensors and biomedical applications [17].

\section{Acknowledgements}

This work was supported by FCT-Portugal under the contracts PTDC/CTM/099452/2008, PTDC/CTM/102144/2008 and PEst-OE/EEI/LA0008/2013. QF thanks FCT for a postdoc grant.

\section{References}

[1] Irimia-Vladu M., Saricifcti N. S., Bauer S.: Exotic materials for bio-organic electronics. Journal of Materials Chemistry, 21, 1350-1361 (2011).

DOI: $10.1039 /$ C0JM02444A

[2] Siegel A. C., Phillips S. T., Dickey M. D., Lu N., Suo Z., Whitesides G. M.: Foldable printed circuit boards on paper substrates. Advanced Functional Materials, 20, 28-35 (2010).

DOI: $10.1002 / \mathrm{adfm} .200901363$

[3] Tobjörk D., Österbacka R.: Paper electronics. Advanced Materials, 23, 1935-1961 (2011).

DOI: $10.1002 / \mathrm{adma} .201004692$

[4] Rinaudo M.: Chitin and chitosan: Properties and applications. Progress in Polymer Science, 31, 603-632 (2006).

DOI: 10.1016/j.progpolymsci.2006.06.001 
[5] Pillai C. K. S., Paul W., Sharma C. P.: Chitin and chitosan polymers: Chemistry, solubility and fiber formation. Progress in Polymer Science, 34, 641-678 (2009). DOI: $10.1016 /$ j.progpolymsci.2009.04.001

[6] Singh R., Verma R., Kaushik A., Sumana G., Sood S., Gupta R. K., Malhotra B. D.: Chitosan-iron oxide nano-composite platform for mismatch-discriminating DNA hybridization for Neisseria gonorrhoeae detection causing sexually transmitted disease. Biosensors and Bioelectronics, 26, 2967-2974 (2011). DOI: $10.1016 /$ j.bios.2010.11.047

[7] Smitha B., Sridhar S., Khan A. A.: Polyelectrolyte complexes of chitosan and poly(acrylic acid) as proton exchange membranes for fuel cells. Macromolecules, 37, 2233-2239 (2004). DOI: $10.1021 / \mathrm{ma} 0355913$

[8] Irimia-Vladu M., Troshin P. A., Reisinger M., Shmygleva L., Kanbur Y., Schwabegger G., Bodea M., Schwödiauer R., Mumyatov A., Fergus J. W., Razumov V. F., Sitter H., Sariciftci N. S., Bauer S.: Biocompatible and biodegradable materials for organic field-effect transistors. Advanced Functional Materials, 20, 40694076 (2010).

DOI: $10.1002 / \mathrm{adfm} .201001031$

[9] Zhong C., Deng Y., Roudsari A. F., Kapetanovic A., Anantram M. P., Rolandi M.: A polysaccharide bioprotonic field-effect transistor. Nature Communications, 2, 476/1-476/5 (2011).

DOI: $10.1038 /$ ncomms 1489

[10] Zhou B., Sun J., Han X., Jiang J., Wan Q.: Low-voltage organic/inorganic hybrid transparent thin-film transistors gated by chitosan-based proton conductors. IEEE Electron Device Letters, 32, 1549-1551 (2011). DOI: 10.1109/LED.2011.2164612

[11] Cunha A. G., Fernandes S. C. M., Freire C. S. R., Silvestre A. J. D., Pascoal Neto C., Gandini A.: What is the real value of chitosan's surface energy? Biomacromolecules, 9, 610-614 (2008).

DOI: $10.1021 / \mathrm{bm} 701199 \mathrm{~g}$
[12] Nogales A., Ezquerra T. A., Rueda D. R., Martinez F., Retuert J.: Influence of water on the dielectric behaviour of chitosan films. Colloid and Polymer Science, 275, 419-425 (1997). DOI: $10.1007 / \mathrm{s} 003960050099$

[13] Chu C-W., Li S-H., Chen C-W., Shrotriya V., Yang Y.: High-performance organic thin-film transistors with metal oxide/metal bilayer electrode. Applied Physics Letters, 87, 193508/1-193508/3 (2005).

DOI: $10.1063 / 1.2126140$

[14] Chang J-F., Sun B., Breiby D. W., Nielsen M. N., Sölling T. I., Giles M., McCulloch I., Sirringhaus H.: Enhanced mobility of poly(3-hexylthiophene) transistors by spin-coating from high-boiling-point solvents. Chemistry of Materials, 16, 4772-4776 (2004).

DOI: $10.1021 / \mathrm{cm} 049617 \mathrm{~W}$

[15] Sirringhaus H., Wilson R. J., Friend R. H., Inbasekaran M., Wu W., Woo E. P., Grell M., Bradley D. D. C.: Mobility enhancement in conjugated polymer fieldeffect transistors through chain alignment in a liquidcrystalline phase. Applied Physics Letters, 77, 406408 (2000). DOI: $10.1063 / 1.126991$

[16] Kaihovirta N., Aarnio H., Wikman C-J., Wilén C-E., Österbacka R.: The effects of moisture in low-voltage organic field-effect transistors gated with a hydrous solid electrolyte. Advanced Functional Materials, 20, 2605-2610 (2010). DOI: $10.1002 / \mathrm{adfm} .201000586$

[17] Kim D-H., Viventi J., Amsden J. J., Xiao J. Vigeland L., Kim Y-S., Blanco J. A., Panilaitis B., Frechette E. S., Contreras D., Kaplan D. L., Omenetto F. G., Huang Y., Hwang K-C., Zakin M. R., Rogers J. A.: Dissolvable films of silk fibroin for ultrathin conformal biointegrated electronics. Nature Materials, 9, 511-517 (2010).

DOI: $10.1038 / \mathrm{nmat} 2745$ 